\title{
A Pilot Study on Patterns of Skill Development of Neurotypical Children as Measured by the $A B L L S$ - $R$ : Implications for Educational Programming for Children With Autism
}

\author{
James W. Partington ${ }^{1}$, Autumn Bailey ${ }^{1}$, Scott W. Partington ${ }^{1}$ \\ ${ }^{1}$ James W. Partington, Autumn Bailey, and Scott W. Partington, Pleasant Hill, California, USA \\ Correspondence: James W. Partington, Behavior Analysts. Inc., 3478 Buskirk Ave., Suite 1000, Pleasant Hill, CA 94523, \\ USA.
}

Received: September 13, 2018

Accepted: October 25, $2018 \quad$ Online Published: October 29, 2018

doi:10.11114/ijce.v1i2.3619

URL: https://doi.org/10.11114/ijce.v1i2.3619

\begin{abstract}
Many assessments used to measure the skills of children with an autism spectrum disorder (ASD) diagnosis lack data that delineates patterns of skill development by neurotypical children. In the current pilot study, we administered the Assessment of Basic Language and Learning Skills-Revised (ABLLS-R) to neurotypical children $(N=53)$ between the ages of six and 72 months to examine typical skill development across the major skill sets and repertoires of the $A B L L S-R$. We found that skills from the Basic Learner skills section emerged and developed earlier (i.e., by age five) than those from the other skills sections. By age six, children mastered their motor skills and $90 \%$ of the self-help skills. Academic skills took the longest to emerge and develop with children mastering only $51 \%$ of the related skills by age six. Implications of these findings, as they relate to skill development and identifying developmentally appropriate teaching objectives for individuals with ASD, are discussed.
\end{abstract}

Keywords: autism, $A B L L S-R$, developmental disorders, normative data, applied behavior analysis, verbal behavior

\section{Introduction}

The prevalence of individuals with an autism spectrum disorder (ASD) has drastically increased and now affects one in 59 children (Center for Disease Control and Prevention, 2018). This statistic demonstrates the increasing need for effective educational services and teaching strategies. Individuals tasked with the delivery of such services (e.g., educators, behavior analysts, parents, etc.) should aim to develop and consistently engage in best practice. Guldberg (2010) identified and described several factors that characterize best practice including the ability to recognize the significant differences across individuals with ASD with regard to their skills, intellect, and behavior. Further, she noted the importance of carefully and thoroughly assessing the skills of each individual and tailoring subsequent interventions to meet his or her unique needs. Taken together, one can deduce that engaging in best practice involves the administration of a comprehensive skills assessment to thoroughly measure an extensive range of skills, the application of effective and developmentally appropriate teaching strategies, and tracking client progress over time to increase the likelihood of optimal learning and development. Consequently, practitioners will need to administer a skills assessment that addresses these considerations.

Those seeking to measure the skills of individuals with ASD will typically administer either a norm-referenced assessment or a criterion-referenced assessment. Norm-referenced assessments yield scores that reflect the extent that an individual can perform a specific skill set in relation to his or her same-aged, typically developing peers (e.g., Vineland II; Sparrow, Cicchetti, \& Balla, 2005a) whereas criterion-referenced assessments (e.g., Verbal Behavior Milestones Assessment and Placement Program; Sundberg, 2008) measure the extent that an individual can perform specific skills as measured by his or her ability to meet the stated scoring criteria for each item in the assessment. Thus, scores obtained from the former emphasize inter-individual variability whereas the latter highlights the extent of the unique skills of that individual.

Despite their widespread use, both types of assessments contain, at best, an insufficient amount of empirical research on typical skill development. Consider that most norm-referenced assessments contain data that reflects the performance of neurotypical children across major skill sets and broad subdomains (e.g., Communication, Academics, etc.) at a given 
age. However, these data do not extend to specific skill areas (i.e., repertoires; e.g., Labeling, Requesting, etc.) and the individual skills that comprise them. Thus, educators will likely struggle to identify specific repertoires and more importantly, the specific skills within those repertoires that an individual needs to develop. In stark contrast, criterion-referenced assessments review an extensive range of individual skills across several different repertoires, but they do not contain data that delineates patterns of typical skill development. This limitation results in uncertainty as to the extent that an individual should possess a specific skill at a given age. Regardless of the type of assessment administered, both contain insufficient data on typical skill development-a limitation that leaves educators with minimal and often, inadequate resources for determining developmentally appropriate teaching objectives. An ideal assessment would thoroughly measure the extent (i.e., criterion-referenced assessment) that one possesses various skill sets (e.g., Language), including the repertoires (e.g., Requesting, Labeling, Receptive Language, Intraverbals, etc.) and individual skills that comprise them, and contain skill development data that allows for one to compare the data obtained to that of a same-aged, neurotypical peer.

A popular criterion-referenced assessment tool that yields a comprehensive overview of several essential skills and could prove useful as a means to measure skill development and inform educational programming is the Assessment of Basic Language and Learning Skills-Revised (ABLLS-R; Partington 2010a). Indeed, leading researchers in the field of behavior analysis and several professional organizations identified the $A B L L S-R$ as an important resource and skills tracking system that can guide parents and professionals with teaching critical language and learner skills to children with ASD (Aman et al., 2004; American Medical Association, 2014; Schwartz, Boulware, McBride, \& Sandall, 2001; Thompson, 2011). This widely used assessment reviews 544 skills across 25 different repertoires and emphasizes language, social interaction, self-help, academic, and motor skills. Despite its popularity, a noteworthy limitation of the $A B L L S-R$ includes a lack of data that delineates typical skill development as measured by the specific skills, repertoires, and skill sets that comprise the assessment.

Using the $A B L L S-R$ to delineate patterns of typical skill development can highly benefit those that teach skills to children with ASD while also addressing a prevailing gap in the assessment literature. Using the $A B L L S-R$ to identify patterns of typical development can greatly and positively impact the process of educational programming for parents and educators. Specifically, these data may facilitate the process of determining developmentally appropriate teaching objectives for children with ASD. For example, one might consult skill development data to determine which repertoires to teach first and the specific skills within those repertoires to target, given the age and the existing skills of the student with ASD. In addition to potentially benefitting parents and educators, the present pilot study also greatly contributes to the assessment literature as it marks the first known effort toward establishing developmental norms using a criterion-referenced assessment.

\section{Method}

\subsection{Participants}

\subsubsection{Assessors}

We recruited participants from six-hour training seminars, held in both the United States and Asia, on how to conduct an $A B L L S-R$ skills assessment (i.e., how to administer the assessment, how to score responses, collect data, etc.) delivered by the first author. Following the seminar, some attendees (i.e., parents and professionals), which we will refer to from here forth as "assessors," volunteered to include their typically developing children in our research. All assessors $(N=$ 42 ) received at least a Bachelor level degree and $83 \%$ previously administered the $A B L L S-R$ prior to participating in our study. The assessors did not receive compensation for their participation, however we provided them with free access to their online $A B L L S-R$ account (i.e., WebABLLS) for the duration of the study so that they could enter their data and monitor the development of their child(ren).

\subsubsection{Children}

Upon receiving signed consent forms from the assessors, the researchers inquired about the health of all children enrolled in the study ( $N=53 ; 30$ girls and 23 boys, age range: six to 72 months). All assessors reported their children as healthy and as absent of any mental health disorders or learning disabilities. We then administered the Vineland II questionnaire (Sparrow et al., 2005a) to all of the children to measure their adaptive functioning and found that the vast majority of their Adaptive Behavior Composite scores ( $M=110$, range: 86-131) fell within the normal range.

\subsection{Materials}

\subsubsection{Vineland II}

The Vineland II is a norm-referenced assessment that measures personal and social skills, from birth through adulthood. Scores obtained from the Vineland II reflect the performance of an individual in relation to the performance of a same-aged, typically developing peer. The assessment contains five major domains including Communication, Daily 
Living Skills, Socialization, Motor Skills, and a Maladaptive Behavior Index with each domain containing up to three subdomains. It contains a wealth of empirical support for its pyschometric properties, including various forms of validity and reliability (Sparrow, Cicchetti, \& Balla, 2005b).

\subsubsection{WebABLLS}

The online version of the $A B L L S-R$ (i.e., WebABLLS) is identical to the printed version of the ABLLS-R and contains 25 repertoires - each of which corresponds to one of the following four major sections referenced in the ABLLS-R Scoring Instructions and IEP Development Guide (Partington 2010b): Basic Learner skills, Academic skills, Self-help skills, and Motor skills. The assessment contains a total of 544 items with each repertoire containing between six and 57 ABLLS-R items. Participants can earn a score ranging between zero and up to four points per item depending on the specific scoring criteria for each $A B L L S-R$ item. Its international use by both parents and professionals reflect the strong clinical significance of the assessment and recent empirical evidence points to the ABLLS-R as a valid assessment tool that yields reliable scores (Malkin, Dixon, Speelman, \& Luke, 2016; Partington, Bailey, \& Partington, 2016; Usry, Partington, \& Partington, 2017).

\subsection{Setting and Procedure}

The description provided in the ABLLS-R Scoring Instructions and IEP Development Guide (Partington 2010b) noted that any person familiar with the child could administer the assessment. Further, our recruitment method ensured that all assessors received training on how to administer the $A B L L S-R$, score responses, and collect data. With these considerations in mind, we allowed assessors to independently collect data on the performance of their child (i.e., we did not collect data on inter-observer agreement [IOA] nor did we obtain data on procedural fidelity).

Data collection occurred between January of 2007 and May of 2013 in the home setting. Assessors could initiate data collection as early as six months of age (or at a later age depending on the age of their child) and continue through up to age 72 months. We asked assessors to begin collecting data once the child reached the next three-month stage in development. For example, a parent of a 22-month old child would begin collecting data once their child turned two (i.e., 24 months). At each three-month interval thereafter (e.g., 27 months, 30 months, etc.), participants would receive a two-week data collection window, the week prior to and following the day of the three-month mark, to enter data into their WebABLLS account. The researchers determined that administering the assessment on a frequent basis (i.e., every three months) would enable a more thorough examination of skill development and that two weeks should provide the assessors with ample time to complete the assessment. We informed participants that we scored data obtained outside the two-week window, and incomplete data obtained within the two-week window, as missing data across all repertoires for that data collection period. We encouraged the collection of data through age six, but participants could terminate data collection at their discretion at any time during the study.

\subsection{Data Analysis}

\subsubsection{Dependent Variables}

The present study marks the first known effort to study skill development demonstrated by neurotypical children using the $A B L L S-R$. Thus, we established and calculated two dependent variables to monitor the development of all 25 $A B L L S-R$ repertoires and each of the four major skill sections: the percentage of only fully completed $A B L L S-R$ items and the percentage of both the fully and partially completed $A B L L S-R$ items. The percentage of only fully completed items reflects the extent that children mastered the skills associated with the repertoire or section whereas the percentage of the fully and partially completed items reflect the extent that skills from a given repertoire or section had emerged.

In order to obtain measures of our dependent variables at each age interval, we first needed to take a preliminary step and determine the age that typically developing children mastered each level of scoring criteria for each $A B L L S-R$ item. To complete this precursor step, we established a priori that we considered a scoring level of an $A B L L S-R$ item as "mastered" at the earliest age that $95 \%$ or more of the participant sample met the stated scoring criteria for that particular scoring level of the $A B L L S-R$ item. This conservative mastery criterion, in addition to making calculations at each three-month interval, allowed for a more accurate estimation of the age that our participant sample mastered each level of scoring criteria for each $A B L L S-R$ item in the assessment. To better illustrate our methodology, consider that the $A B L L S-R$ item D9 contains two possible points - a child receives one point for imitating one head movement and two points for imitating three or more head movements. This preliminary step in the analysis yielded data on the earliest age that $95 \%$ (or more) of our participant sample met the scoring criteria for one point and two points for this specific $A B L L S$ - $R$ item.

After determining the age that participants mastered the criteria for each scoring level for each $A B L L S-R$ item, we used those data to obtain measures of our two dependent variables at each age interval. To accomplish this multi-step task, 
we first coded all $A B L L S-R$ items from each repertoire and section as either "partially complete" or "fully complete" at each age interval. Partially complete $A B L L S-R$ items consist of those in which $95 \%$ or more of the sample scored at least a one, but did not meet the scoring criteria for the highest possible score. We consider an $A B L L S-R$ item as "mastered" when at least $95 \%$ of the participant sample met the scoring criteria for the highest possible score. One exception to this coding method includes the $A B L L S-R$ items from the Fine and Gross Motor repertoires as these items contain a maximum of one possible point per item. Consequently, we only examined the mastery of these skills by calculating the percentage of fully complete items from these repertoires as well as the Motor skills section.

Following the process of coding all applicable $A B L L S-R$ items as fully or partially complete, we obtained measures of our two dependent variables for all $25 A B L L S-R$ repertoires and each of the four major sections, at each age interval. To obtain the percentage of fully complete items from an $A B L L S-R$ repertoire or section at a given age, we calculated the sum of the fully complete items from the repertoire or section of interest, divided that number by the total number of items that comprise it, and multiplied the quotient by 100. For example, to calculate the percentage of only fully complete $A B L L S-R$ items for the Basic Learner section (i.e., repertoires A-P) at a given age, obtain the sum of the fully complete items from repertoires A-P, divide the sum by the total number of items that comprise this section (i.e., 381 items in repertoires A-P), and multiply the quotient by 100 . To calculate the percentage of both the fully and partially complete items at a given age, we obtained the sum of the fully and partially complete $A B L L S-R$ items from the repertoire or section of interest, divided the sum by the total number of items that comprise the repertoire or section, and multiplied the quotient by 100 . We consider $A B L L S-R$ repertoires and sections as "mastered" when $95 \%$ or more of its items are coded as "fully complete."

\section{Results}

We took a preliminary step prior to examining the emergence and development of skills from each section of the $A B L L S-R$ and its corresponding repertoires by identifying the age that $95 \%$ or more of our participant sample met each level of scoring criteria for each $A B L L S-R$ item. We provided visual displays depicting our results for all $A B L L S-R$ items in repertoires A through I (see Figure 1), J through R (see Figure 2), and S through Z (see Figure 3) with the number inside each cell representing the age that $95 \%$ or more of the typically developing children met the stated scoring criteria for that particular score. Note that in these figures, blank cells reflect scoring criteria that the children did not master by age six or for one specific item (i.e., H3), it represents a skill that is not generally used by typically developing children (i.e., intraverbal sign language). Although there are some exceptions, we also observed a general pattern of skill development that showed that children typically mastered the lower numbered $A B L L S-R$ skills (e.g., F1-F6) at an earlier age than the higher numbered skills (e.g., F22-F29). The data obtained from this preliminary step enabled us to calculate the extent of repertoire and major skill section development in the subsequent analyses. 


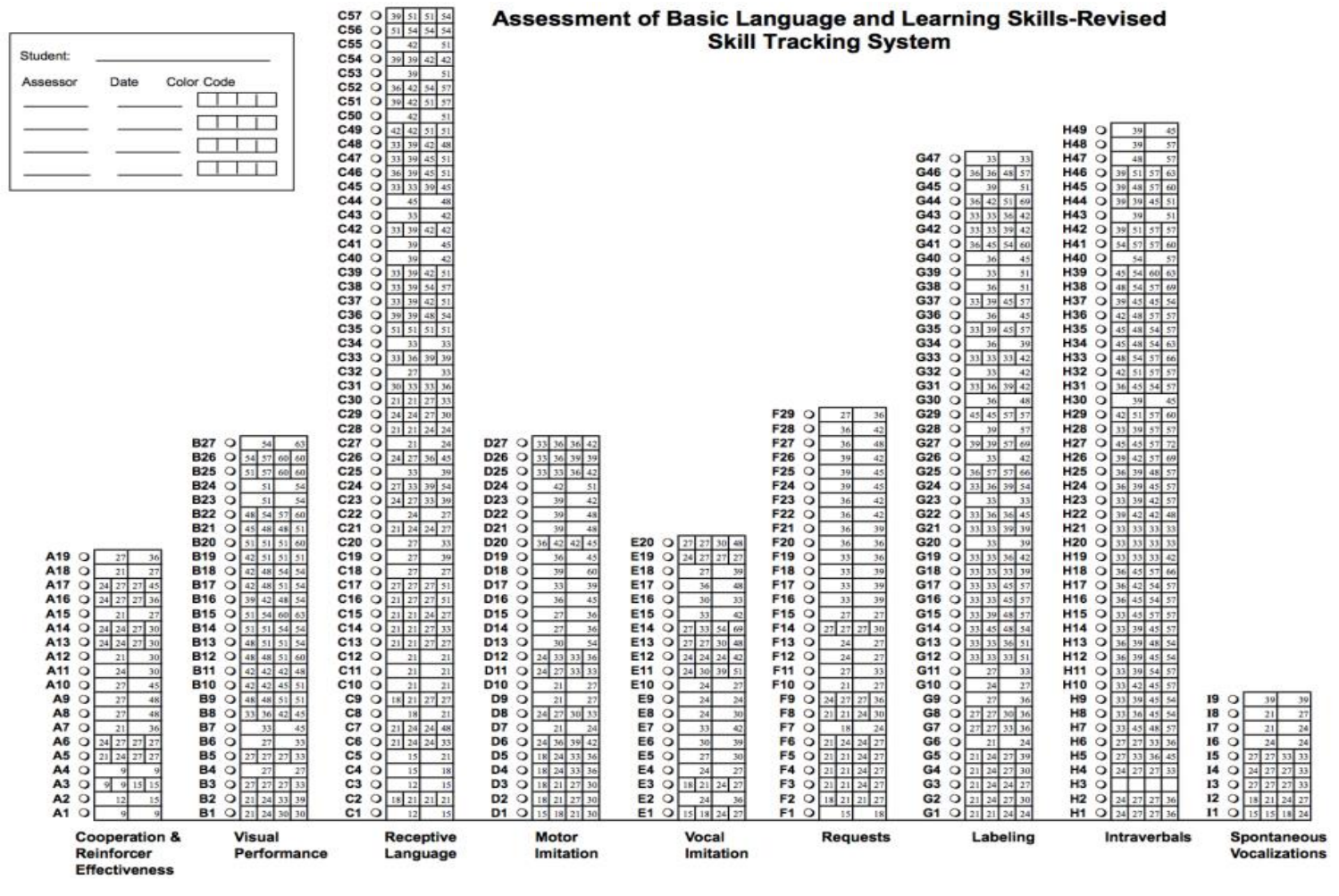

Figure 1. Mastery of the scoring criteria (in months) for ABLLS-R items in repertoires A through I 


\section{Assessment of Basic Language and Learning Skills-Revised \\ Skill Tracking System}
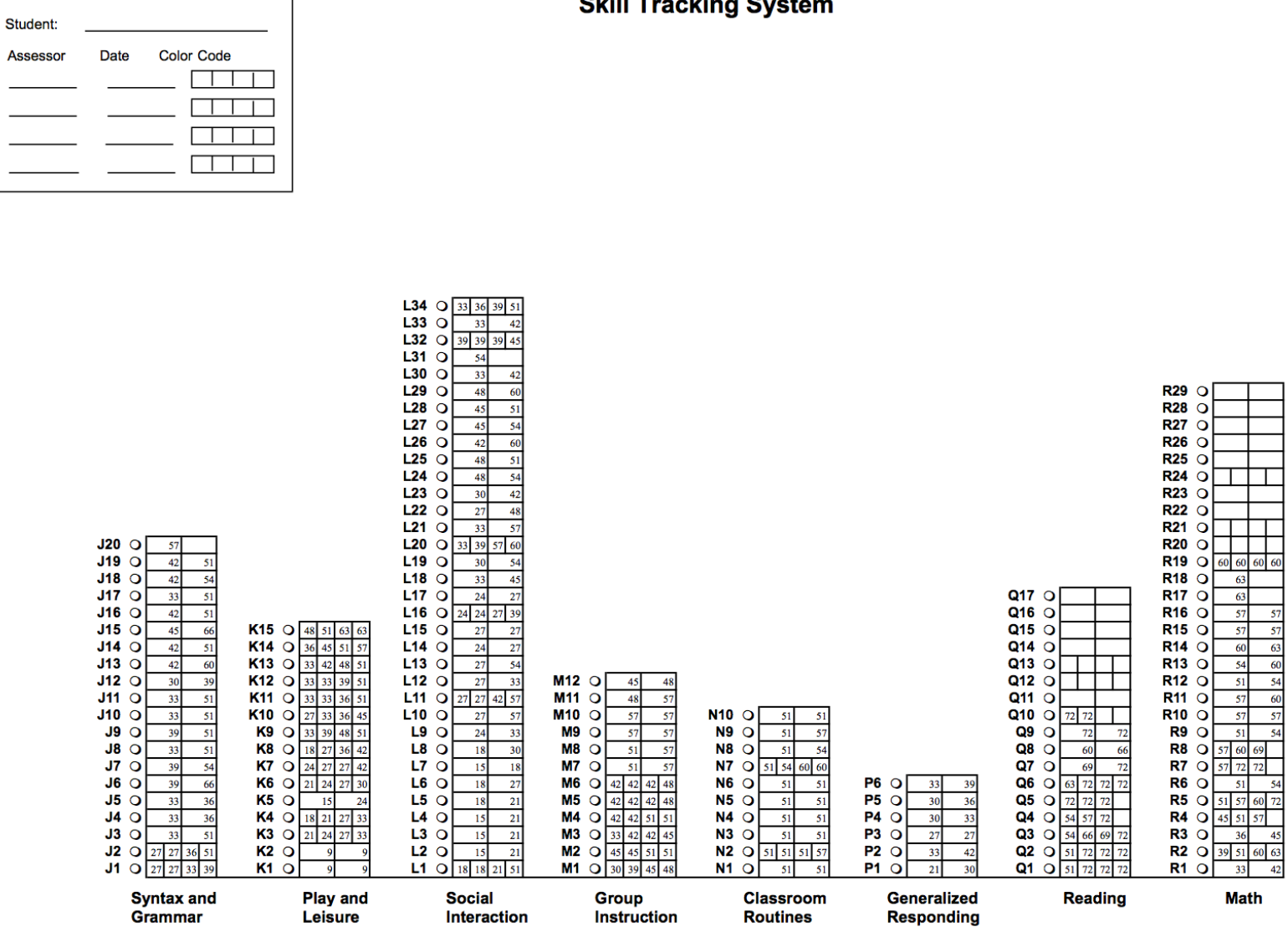

Figure 2. Mastery of the scoring criteria (in months) for ABLLS-R items in repertoires $\mathrm{J}$ through $\mathrm{R}$ 
Assessment of Basic Language and Learning Skills-Revised

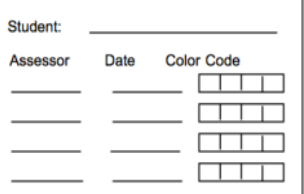

Skill Tracking System

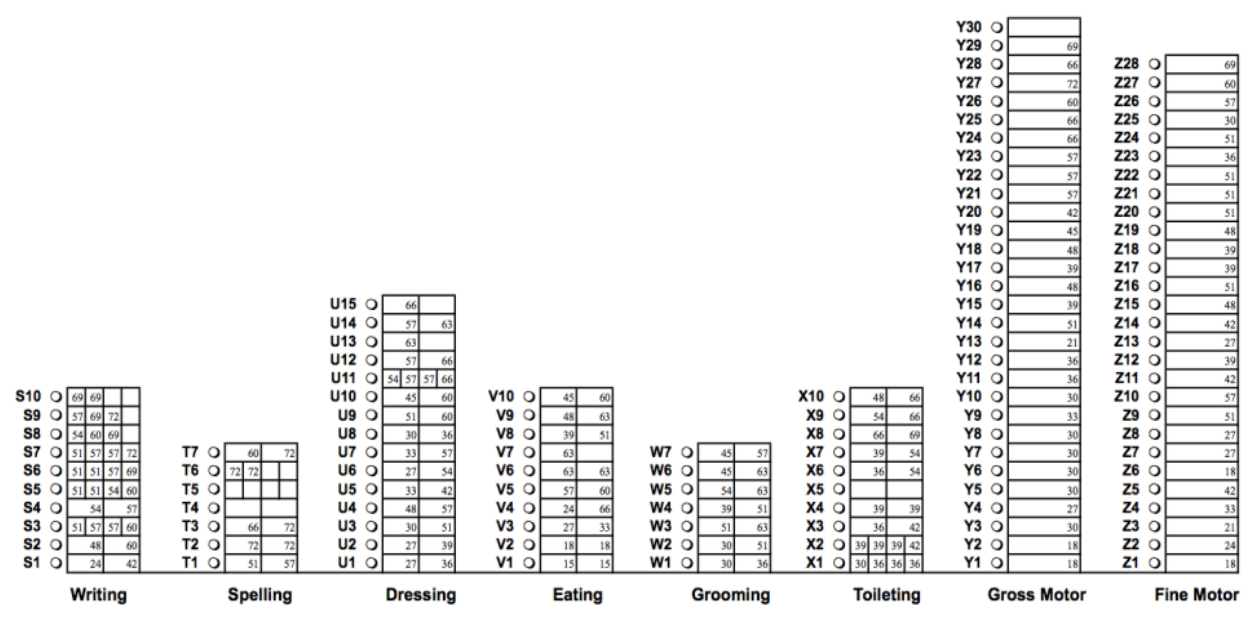

Figure 3. Mastery of the scoring criteria (in months) for ABLLS-R items in repertoires $\mathrm{S}$ through $\mathrm{Z}$

\subsection{Emergence and Mastery of ABLLS-R Repertoires}

When measuring the development of skills from each major skills section and repertoire from the ABLLS-R, we used data obtained from the preliminary step in the analysis to obtain measures of our two dependent variables. We then graphed these data at ages six $(n=12), 12(n=18), 18(n=24), 24(n=32), 30(n=29), 36(n=32), 42(n=34), 48(n$ $=31), 54(n=25), 60(n=25), 66(n=13)$, and 72 months $(n=13)$ for each repertoire and major skill section from the assessment. These data revealed patterns of typical skill development across the ABLLS-R repertoires, including the extent that each repertoire emerged (see Table 1) and the percentage of the repertoire mastered (see Table 2), at each six-month age interval. 
Table 1. The Percentage of Skills Emerged from Each ABLLS-R Repertoire

\begin{tabular}{|c|c|c|c|c|c|c|c|c|c|c|c|c|}
\hline Repertoire Label & $\begin{array}{c}.5 \\
\text { Yrs }\end{array}$ & $\begin{array}{c}1 \\
\text { Yrs }\end{array}$ & $\begin{array}{l}1.5 \\
\text { Yrs }\end{array}$ & $\begin{array}{c}2 \\
\text { Yrs }\end{array}$ & $\begin{array}{l}2.5 \\
\text { Yrs }\end{array}$ & $\begin{array}{c}3 \\
Y r s\end{array}$ & $\begin{array}{l}3.5 \\
\text { Yrs }\end{array}$ & $\begin{array}{c}4 \\
\text { Yrs }\end{array}$ & $\begin{array}{l}4.5 \\
\text { Yrs }\end{array}$ & $\begin{array}{c}5 \\
\text { Yrs }\end{array}$ & $\begin{array}{l}5.5 \\
\text { Yrs }\end{array}$ & $\begin{array}{c}6 \\
\text { Yrs }\end{array}$ \\
\hline $\begin{array}{l}\text { A Cooperation \& Reinforcer } \\
\text { Effectiveness }\end{array}$ & 0 & 21 & 21 & 79 & 100 & 100 & 100 & 100 & 100 & 100 & 100 & 100 \\
\hline B Visual Performance & 0 & 0 & 0 & 7 & 22 & 30 & 52 & 78 & 100 & 100 & 100 & 100 \\
\hline C Receptive Language & 0 & 3 & 12 & 42 & 54 & 77 & 95 & 96 & 100 & 100 & 100 & 100 \\
\hline D Motor Imitation & 0 & 0 & 19 & 44 & 56 & 81 & 100 & 100 & 100 & 100 & 100 & 100 \\
\hline E Vocal Imitation & 0 & 0 & 10 & 50 & 85 & 100 & 100 & 100 & 100 & 100 & 100 & 100 \\
\hline F Requesting & 0 & 0 & 10 & 41 & 55 & 90 & 100 & 100 & 100 & 100 & 100 & 100 \\
\hline G Labeling & 0 & 0 & 0 & 15 & 23 & 91 & 98 & 100 & 100 & 100 & 100 & 100 \\
\hline $\mathrm{H}$ Intraverbals & 0 & 0 & 0 & 6 & 10 & 52 & 81 & 96 & 100 & 100 & 100 & 100 \\
\hline I Spontaneous Vocalizations & 0 & 0 & 22 & 67 & 89 & 89 & 100 & 100 & 100 & 100 & 100 & 100 \\
\hline J Syntax and Grammar & 0 & 0 & 0 & 0 & 15 & 50 & 90 & 95 & 95 & 100 & 100 & 100 \\
\hline K Play and Leisure & 0 & 13 & 33 & 53 & 60 & 93 & 93 & 100 & 100 & 100 & 100 & 100 \\
\hline L Social Interaction & 0 & 0 & 24 & 35 & 59 & 76 & 82 & 97 & 100 & 100 & 100 & 100 \\
\hline M Group Instruction & 0 & 0 & 0 & 0 & 8 & 17 & 42 & 67 & 83 & 100 & 100 & 100 \\
\hline N Classroom Routines & 0 & 0 & 0 & 0 & 0 & 0 & 0 & 0 & 100 & 100 & 100 & 100 \\
\hline P Generalized Responding & 0 & 0 & 0 & 17 & 67 & 100 & 100 & 100 & 100 & 100 & 100 & 100 \\
\hline Q Reading & 0 & 0 & 0 & 0 & 0 & 0 & 0 & 0 & 24 & 29 & 35 & 59 \\
\hline R Math & 0 & 0 & 0 & 0 & 0 & 7 & 10 & 14 & 31 & 59 & 66 & 66 \\
\hline S Writing & 0 & 0 & 0 & 10 & 10 & 10 & 10 & 20 & 80 & 90 & 90 & 100 \\
\hline T Spelling & 0 & 0 & 0 & 0 & 0 & 0 & 0 & 0 & 14 & 29 & 43 & 86 \\
\hline U Dressing & 0 & 0 & 0 & 0 & 33 & 47 & 47 & 60 & 73 & 87 & 100 & 100 \\
\hline V Eating & 0 & 0 & 20 & 30 & 40 & 40 & 50 & 70 & 70 & 80 & 100 & 100 \\
\hline W Grooming & 0 & 0 & 0 & 0 & 29 & 29 & 43 & 71 & 100 & 100 & 100 & 100 \\
\hline X Toileting & 0 & 0 & 0 & 0 & 10 & 20 & 60 & 70 & 80 & 80 & 90 & 90 \\
\hline
\end{tabular}

Note. The percentages above reflect the percentage of fully and partially complete items (i.e., emerged) from each ABLLS-R repertoire. The items from the Gross $(\mathrm{Y})$ and Fine Motor $(\mathrm{Z})$ repertoires contain a maximum of only one possible point and therefore, cannot be coded as partially complete (and thereby used to measure the emergence of skills from these specific repertoires). 
Table 2. The Percentage of Skills Mastered from Each ABLLS-R Repertoire

\begin{tabular}{|c|c|c|c|c|c|c|c|c|c|c|c|c|}
\hline Repertoire Label & .5 & 1 & 1.5 & 2 & 2.5 & 3 & 3.5 & 4 & 4.5 & 5 & 5.5 & $\begin{array}{c}6 \\
Y r\end{array}$ \\
\hline $\begin{array}{l}\text { A Cooperation \& Reinforcer } \\
\text { Effectiveness }\end{array}$ & 0 & 11 & 21 & 21 & 67 & 79 & 79 & $100^{*}$ & 100 & 100 & 100 & 100 \\
\hline B Visual Performance & 0 & 0 & 0 & 0 & 7 & 19 & 22 & 33 & 74 & 93 & $100 *$ & 100 \\
\hline C Receptive Language & 0 & 0 & 5 & 19 & 32 & 44 & 58 & 68 & $95^{*}$ & 100 & 100 & 100 \\
\hline D Motor Imitation & 0 & 0 & 0 & 4 & 22 & 48 & 70 & 89 & $96^{*}$ & 100 & 100 & 100 \\
\hline E Vocal Imitation & 0 & 0 & 0 & 5 & 40 & 50 & 75 & 90 & $95^{*}$ & 95 & 95 & 100 \\
\hline F Requesting & 0 & 0 & 3 & 7 & 45 & 62 & 90 & $100 *$ & 100 & 100 & 100 & 100 \\
\hline G Labeling & 0 & 0 & 0 & 4 & 13 & 26 & 49 & 60 & 74 & 94 & $96^{*}$ & 100 \\
\hline $\mathrm{H}$ Intraverbals & 0 & 0 & 0 & 0 & 0 & 13 & 15 & 23 & 38 & 83 & 94 & $100^{*}$ \\
\hline I Spontaneous Vocalizations & 0 & 0 & 0 & 33 & 56 & 89 & $100 *$ & 100 & 100 & 100 & 100 & 100 \\
\hline J Syntax and Grammar & 0 & 0 & 0 & 0 & 0 & 10 & 15 & 20 & 80 & 85 & $95^{*}$ & 95 \\
\hline K Play and Leisure & 0 & 13 & 13 & 20 & 27 & 40 & 53 & 60 & 87 & 93 & $100 *$ & 100 \\
\hline L Social Interaction & 0 & 0 & 3 & 15 & 29 & 35 & 44 & 56 & 79 & $97 *$ & 97 & 97 \\
\hline M Group Instructions & 0 & 0 & 0 & 0 & 0 & 0 & 0 & 42 & 58 & $100^{*}$ & 100 & 100 \\
\hline N Classroom Routines & 0 & 0 & 0 & 0 & 0 & 0 & 0 & 0 & 80 & 90 & $100 *$ & 100 \\
\hline P Generalized Responding & 0 & 0 & 0 & 0 & 33 & 67 & $100 *$ & 100 & 100 & 100 & 100 & 100 \\
\hline Q Reading & 0 & 0 & 0 & 0 & 0 & 0 & 0 & 0 & 0 & 0 & 6 & 41 \\
\hline R Math & 0 & 0 & 0 & 0 & 0 & 0 & 3 & 7 & 7 & 38 & 45 & 48 \\
\hline S Writing & 0 & 0 & 0 & 0 & 0 & 0 & 10 & 10 & 10 & 50 & 50 & 70 \\
\hline T Spelling & 0 & 0 & 0 & 0 & 0 & 0 & 0 & 0 & 0 & 14 & 14 & 57 \\
\hline U Dressing & 0 & 0 & 0 & 0 & 0 & 13 & 27 & 27 & 40 & 67 & 80 & 87 \\
\hline V Eating & 0 & 0 & 20 & 20 & 20 & 30 & 30 & 30 & 40 & 60 & 90 & 90 \\
\hline W Grooming & 0 & 0 & 0 & 0 & 0 & 14 & 14 & 14 & 43 & 57 & $100 *$ & 100 \\
\hline X Toileting & 0 & 0 & 0 & 0 & 0 & 10 & 40 & 40 & 60 & 60 & 80 & 90 \\
\hline Y Gross Motor & 0 & 0 & 7 & 10 & 33 & 43 & 53 & 63 & 67 & 80 & 90 & $97 *$ \\
\hline Z Fine Motor & 0 & 0 & 4 & 14 & 29 & 36 & 57 & 64 & 86 & $96^{*}$ & 96 & 100 \\
\hline
\end{tabular}

Note. The percentages above reflect the percentage of fully complete items (i.e., mastered) from each $A B L L S-R$ repertoire. An asterisk denotes the earliest age that children mastered at least $95 \%$ of the items from its corresponding repertoire.

\subsubsection{The Emergence and Mastery of ABLLS-R Repertoires From the Basic Learner Skills Section}

The repertoires from the Basic Learner skills section emerged at an earlier age than those from the other $A B L L S-R$ sections. As shown in Table 1, skills from most of the repertoires (i.e., 80\%) emerged by age two including skills from the Cooperation and Reinforcer Effectiveness, Receptive Language, Play and Leisure, Motor Imitation, Vocal Imitation, Requesting, Spontaneous Vocalization, Social Interaction, Visual Performance, Labeling, Intraverbals, and Generalized Responding repertoires. The early emergence of skills from these repertoires coincides with the finding that typically developing children also mastered many of the skills from the repertories in the Basic Learner skills section at an early age (see Table 2). Specifically, typically developing children mastered $60 \%$ of the repertories from the Basic Learner skills section by age five, $93 \%$ by age five and a half, and all of the repertoires from this skills section by age six.

The developmental path of most of the repertoires from this skill section was characterized by evidence of emergence at a very early age and achieving full (or close to full) development by around age five. Using the data from Tables 1 and 2, we provided a graphical display of the development of the Receptive Language repertoire (see Figure 4), as it reflects commonly observed patterns of typical skill development across several of the repertoires within this particular skill section. One can obtain graphical displays for each repertoire in the $A B L L S-R$ by contacting the first author. 


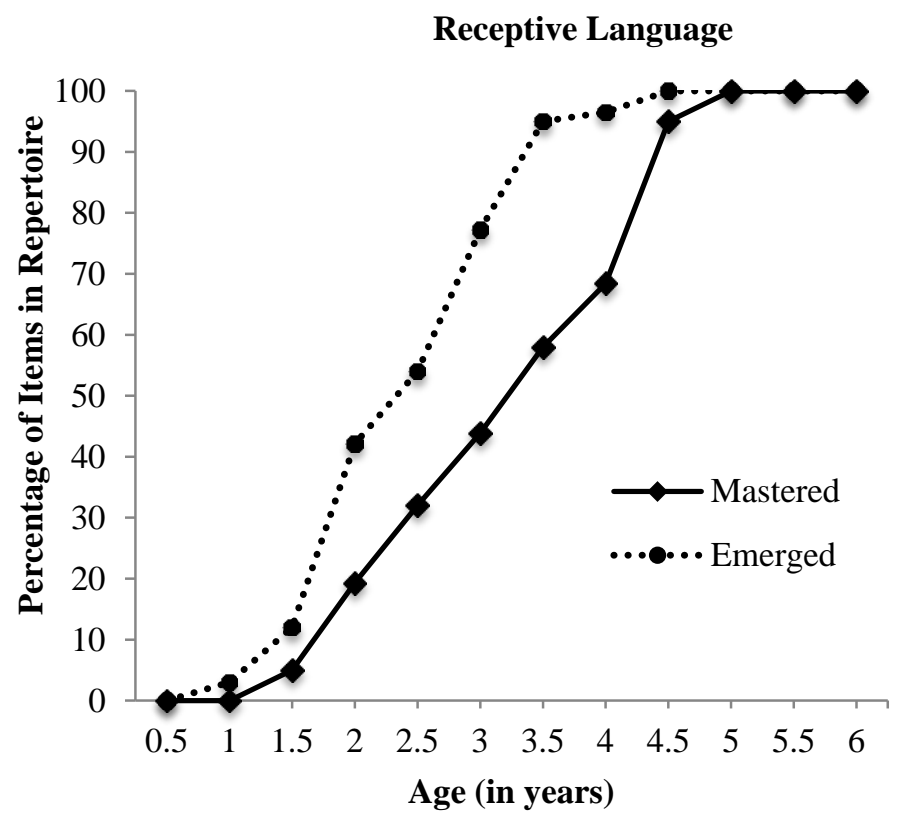

Figure 4. The development of the Receptive Language repertoire from the Basic Learner skills section of the ABLLS-R

\subsubsection{The Emergence and Mastery of $A B L L S-R$ Repertoires From the Academic Skills Section}

In general, the repertoires from the Academic skills section emerged at a later age than the repertoires from any of the other $A B L L S-R$ sections. Only one skill from the Writing repertoire emerged by age two (i.e., $A B L L S-R$ item $S 1-$ the student uses any grip to hold a pen or pencil and draw a line)—only a few skills from the Math, Reading, and Spelling repertoires emerged at or after the age of three (see Table 1). Typically developing children also took a longer time to master their academic skills. By age six, children did not display mastery of any of the academic repertoires and further, they mastered less than half of the skills from the Reading and Math repertoires by this age (see Table 2). We provided a graph portraying the development of the Math repertoire (see Figure 5) to better illustrate the patterns of typical skill development associated with the repertoires from this specific skill section.

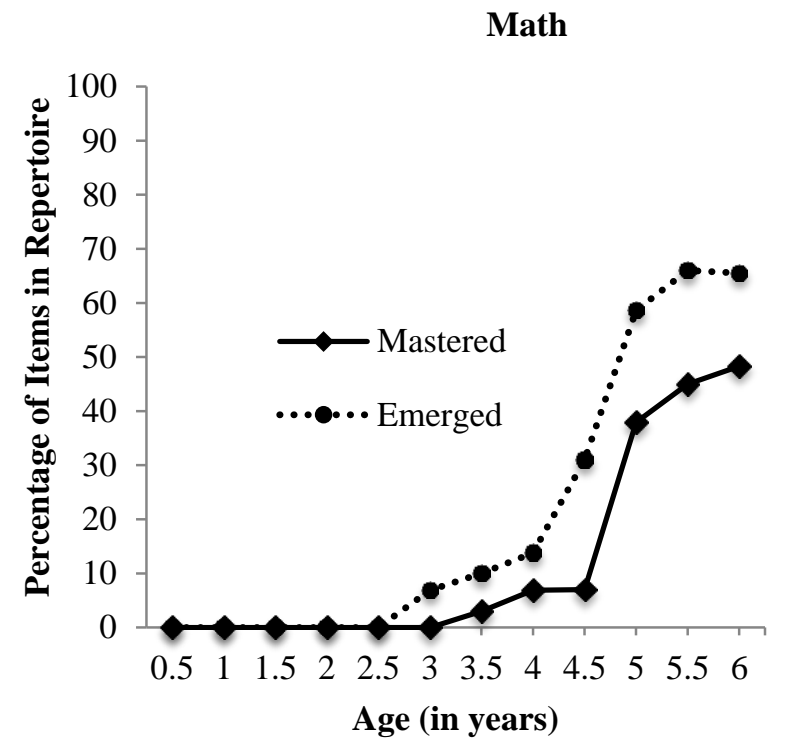

Figure 5. The development of the Math repertoire from the Academic skills section of the ABLLS-R 


\subsubsection{The Emergence and Mastery of $A B L L S-R$ Repertoires From the Self-Help Skills Section}

Similar to the Basic Learner skills section, skills from the repertoires from the Self-help skills section emerged at an early age — skills from each repertoire emerged by age two and a half (see Table 1). We found that despite the noticeable gains made in skill development, children only mastered the skills from the Grooming repertoire by age six (see Table 2). The data provided demonstrate that children mastered several skills from the Dressing (i.e., $87 \%$ of items mastered), Eating (i.e., $90 \%$ of items mastered), and Toileting (i.e., $90 \%$ of items mastered) repertoires, but they did not display mastery over these repertoires by six years of age. The graphical display of the Dressing repertoire reflects the characteristics of many of the self-help repertoires-skills emerged at an early age and children mastered most of the skills from each repertoire by age six (see Figure 6).

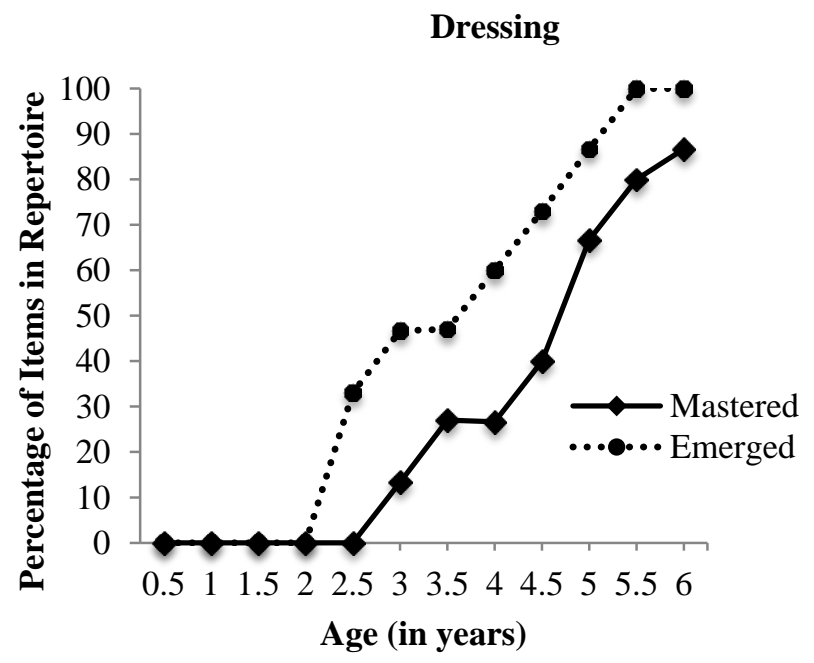

Figure 6. The development of the Dressing repertoire from the Self-help skills section of the ABLLS-R

\subsubsection{Mastery of ABLLS-R Repertoires From the Motor Skills Section}

Our results indicate that children possessed some, albeit minimal gross and fine motor skills by age one and a half. These repertoires appeared to gradually develop to the point that children mastered their fine motor skills by age five and their gross motor skills by age six. The graphical display of the development of the Fine Motor repertoire (see Figure 7) reflects the steady development of these skills.

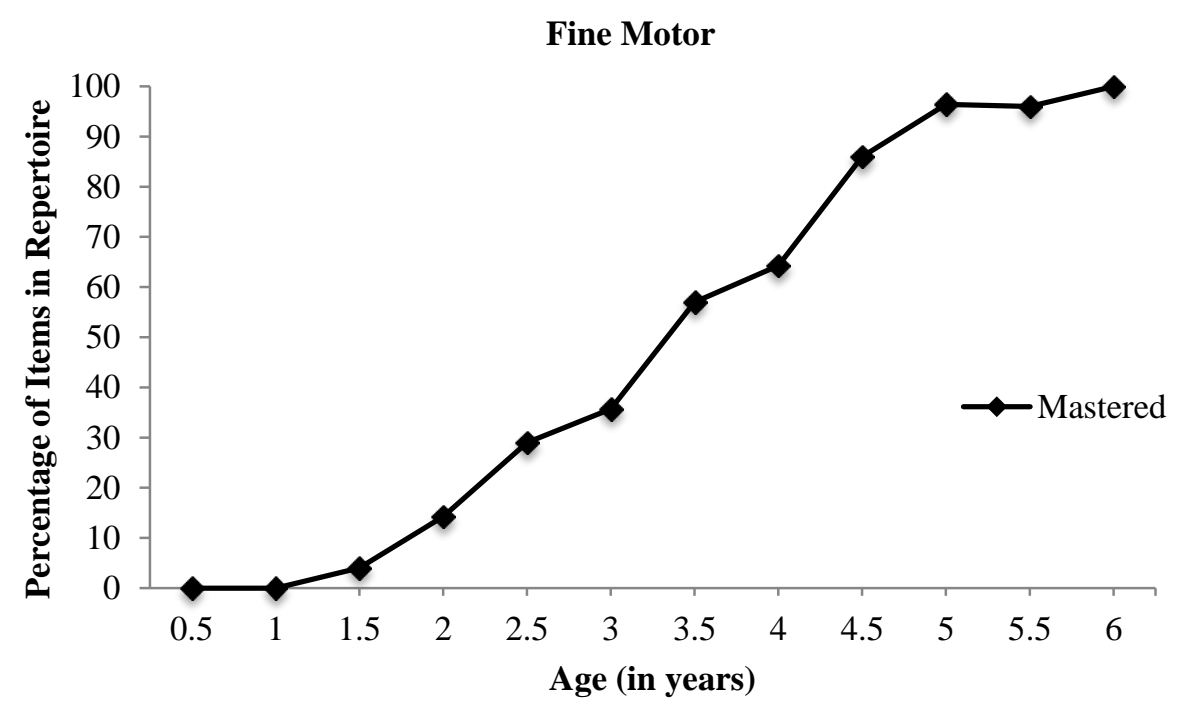

Figure 7. The development of the Fine Motor repertoire from the Motor skills section of the ABLLS-R 


\subsection{Emergence and Mastery of the Major ABLLS-R Sections}

As shown in Table 3, skills from the Basic Learner skills section emerged prior to the skills from the other sections (i.e., by age one). Skills emerged from the Motor skills and Self-help skills sections by age one and a half and skills from the Academic skills section emerged by age two. Although skills from each ABLLS-R section emerged by this age, the overall rate of skill development varied across the four sections. The graphical displays portraying the emergence and mastery of skills from the Basic Learner, Motor, Self-help, and Academic skills sections (see Figure 8) reflect this general finding. The descriptive statics and a visual inspection of the graphs revealed that by age two, the Basic Learner skills section contained the highest percentage of skills emerged (i.e., 29\%) in relation to the Motor (i.e., 12\%), Self-help (i.e., 7\%), and Academic (i.e., 2\%) skills sections. By age four, these percentages increased to 93\%, 64\%, 67\%, and $10 \%$ for each of the aforementioned sections, respectively.

Table 3. The Percentage of Skills Emerged from Each Section of the ABLLS-R

\begin{tabular}{cllllllllllll}
\hline ABLLS-R Section & .5 & 1 & 1.5 & 2 & 2.5 & 3 & 3.5 & 4 & 4.5 & 5 & 5.5 & 6 \\
& $Y r s$ & $Y r$ & Yrs & Yrs & Yrs & Yrs & Yrs & Yrs & Yrs & Yrs & Yrs & Yrs \\
\hline Basic Learner skills & 0 & 2 & 9 & 29 & 43 & 72 & 86 & 93 & 99 & 100 & 100 & 100 \\
Self-help skills & 0 & 0 & 5 & 7 & 29 & 38 & 50 & 67 & 79 & 86 & 98 & 98 \\
Academic skills & 0 & 0 & 0 & 2 & 2 & 5 & 6 & 10 & 35 & 52 & 59 & 70
\end{tabular}

Note. The data in this table reflect the percentage of fully and partially complete items (i.e., emerged) from each $A B L L S-R$ section.
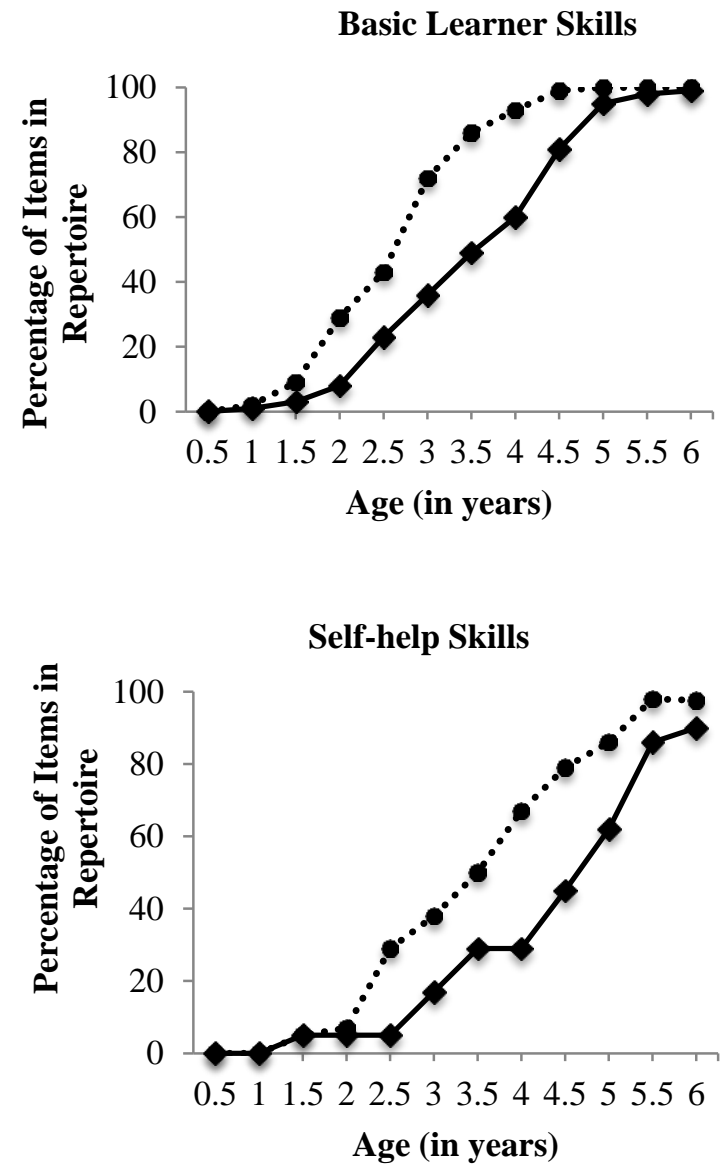
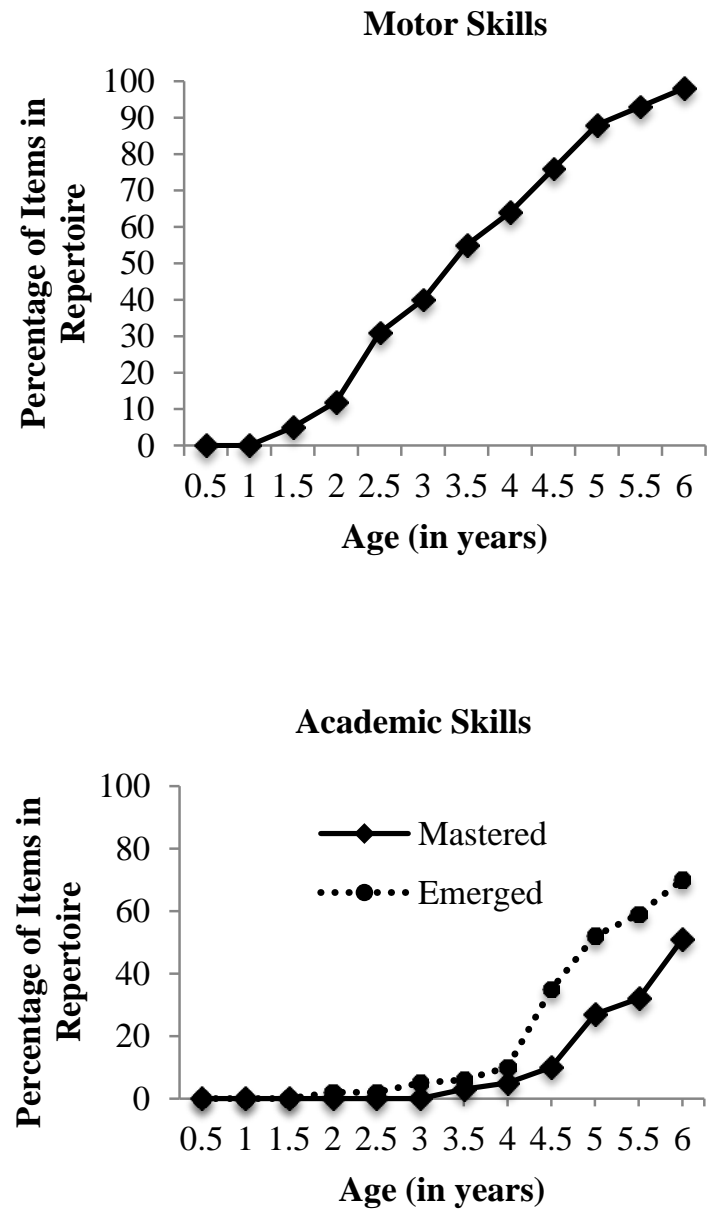

Figure 8. The development of skills from the four major sections of the ABLLS-R 
Children mastered skills from only two of the four $A B L L S-R$ sections by age six (see Table 4). These skills include those from the Basic Learner and Motor skills sections by ages five and six, respectively. In addition, although they did not display mastery over their self-help skills, neurotypical children developed the vast majority (i.e., 90\%) of them by age six. The skills from the Academic section contained the least amount of growth and development through age five in relation to the skills from the other $A B L L S-R$ sections. Our data revealed that children did not master any of the $A B L L S-R$ items from the Academic skills section until age three and a half. Further, our results indicate that typically developing children mastered only 5\% of the $A B L L S-R$ items from the Academic section by age four, slightly over a quarter by age five (i.e., $27 \%$ ), and barely over half (i.e., $51 \%$ ) by age six.

Table 4. The Percentage of Skills Mastered from Each Section of the ABLLS-R

\begin{tabular}{ccccccccccccc}
\hline ABLLS-R Section & .5 & 1 & 1.5 & 2 & 2.5 & 3 & 3.5 & 4 & 4.5 & 5 & 5.5 & 6 \\
& $Y r s$ & $Y r$ & $Y r s$ & $Y r s$ & $Y r s$ & $Y r s$ & $Y r s$ & $Y r s$ & $Y r s$ & $Y r s$ & $Y r s$ & $Y r s$ \\
\hline Basic Learner skills & 0 & 1 & 3 & 8 & 23 & 36 & 49 & 60 & 81 & $95^{*}$ & 98 & 99 \\
Self-help skills & 0 & 0 & 5 & 5 & 5 & 17 & 29 & 29 & 45 & 62 & 86 & 90 \\
Academic skills & 0 & 0 & 0 & 0 & 0 & 0 & 3 & 5 & 10 & 27 & 32 & 51 \\
Motor skills & 0 & 0 & 5 & 12 & 31 & 40 & 55 & 64 & 76 & 88 & 93 & $98^{*}$
\end{tabular}

Note. The data in this table represent the percentage of fully complete items (i.e., mastered) from each ABLLS-R section. The asterisk reflects the earliest age that participants mastered $95 \%$ or more of the items from its corresponding section.

\section{Discussion}

The current pilot study aimed to delineate patterns of skill development demonstrated by neurotypical children so that parents and educators could make data-driven decisions when determining educational priorities for individuals with ASD. To achieve our research objective, we used $A B L L S-R$ data obtained from neurotypical children to identify the age that typically developing children mastered each level of scoring criteria for each $A B L L S-R$ item. These data enabled us to make subsequent calculations and thereby examine the emergence and mastery of skills from each of the four major sections of the $A B L L S-R$ and their corresponding repertoires.

Our research team took an unconventional approach to meet our research objectives. The results obtained from the present study relied on descriptive statistics and visual inspections of graphical displays of our data as opposed to conducting statistical analyses. When deciding upon the appropriate methodology to use, we considered the suggestions provided by Ator (1999) who called for researchers to consider the experimental question, design, and data obtained when determining the appropriateness of inferential statistics. Parameters from the present study, which included our goal of examining and providing visual evidence to illustrate patterns of typical skill development across the different sections of the $A B L L S-R$ and their corresponding repertoires, influenced our decision to forgo the conventional method of using statistical analyses to highlight age-related gains made in skill development. By conducting a visual inspection of the graphical displays and examining the descriptive statistics presented in the tables, readers can observe the obvious changes (or lack thereof in some cases) in the dependent variables as a function of increased age, for each major section and repertoire in the $A B L L S-R$. Furthermore, many researchers have historically encouraged the practice of visually inspecting graphical displays of data as opposed to evaluating results based on data obtained from statistical analyses (e.g., Fahmie \& Hanley, 2008; Perone, 1999; Sidman, 1960).

The resulting patterns of typical skill development afford parents, professionals, and researchers with several benefits. Prior to examining the development of the major $A B L L S-R$ sections and their corresponding repertoires, we first determined the age that $95 \%$ of our participant sample met each level of the scoring criteria for each ABLLS-R item. Findings obtained from this initial step provide a unique contribution to the assessment literature and can highly benefit those who teach skills to children with ASD. Specifically, these data can facilitate the process of selecting developmentally appropriate teaching objectives by shedding light on which $A B L L S-R$ skills to teach a student given their current age and existing skills. For example, if a teacher wanted to teach motor imitation to a 30-month-old learner with ASD, our data reveal that age-appropriate targets may include items D1-D3, D9, D10, and the criteria for the score of a "one" on items D14 and D15 since typically developing children display mastery over these ABLLS-R items and scoring levels by 30 months of age. Likewise, we can use these data to infer that other ABLLS-R items, such as D16-D27 and the criteria for scoring a "two" on items D14 and D15, may not represent developmentally appropriate teaching objectives for a 30-month-old learner with ASD since typically developing children acquire these skills at a later age. Parents or professionals can then use these data to determine what skills to teach and may consider starting 
with skills that typically developing children master at the earliest possible age. For example, they might consider teaching D1 before D2 because typically developing children master the first three scoring levels of the item D1 at an earlier age than D2. While these data can guide program development, we also used them to calculate the descriptive statistics needed to examine the emergence and development of skills from the major ABLLS-R sections and their corresponding repertoires.

A second benefit to examining the emergence and mastery of skills from the major skill sections of the $A B L L S-R$ and their corresponding repertoires includes an enhanced insight into typical skill development. With these data, researchers and practitioners can now determine the extent that typically developing children mastered major skill sections (e.g., Basic Learner skills), repertoires (e.g., motor imitation), and individual skills within those repertoires (e.g., motor imitation using objects, imitation of leg and foot movements, etc.) at any half-year age interval through age six. Parents and professionals can reference these data when identifying skills to teach an individual with ASD. For instance, one can infer from these data that developmentally, one should consider teaching a child with ASD skills from the Receptive Language and Labeling repertoires prior to teaching him or her skills from the Intraverbal repertoire since typically developing children master skills from the former two repertoires at an earlier age than the latter.

One can also use the skill development data obtained from the present study to confirm the appropriateness of programming guidelines and recommendations provided by experts in the area of ASD treatment. Specifically, some popular resources provide general recommendations for educational programming, but fail to document adequate empirical evidence in support of their claims. One such example includes the $A B L L S-R$ guidebook (Partington, 2010b) in which the author strongly recommends teaching skills from the Basic Learner skills section prior to teaching academic skills. Despite the lack of references provided in the guidebook, our results coincide with and confirm the appropriateness of these suggestions. In contrast, our findings do not fully support the description of a curriculum provided by other experts in the area of ASD treatment. When describing the characteristics of a curriculum, Leaf and McEachin (1999) stated, "A strong emphasis should be placed on learning to talk, the development of conceptual and academic skills, and promoting play and social skills. However, as a child gets older, the emphasis should shift to practical knowledge and adaptive skills." While our findings support their recommendation to initially place a strong emphasis on teaching language to children with ASD, they do not support their recommendation that calls for the teaching of conceptual and academic skills prior to adaptive skills.

In addition to facilitating the process of determining developmentally appropriate educational priorities, our data also point to skill development as a complex process that likely includes complex relationships between specific repertoires. Consider the four repertoires from the Basic Learner skills section that developed by age four: Spontaneous Vocalizations, Cooperation and Reinforcer Effectiveness, Requesting, and Generalized Responding. The observation that children displayed noticeable increases or mastered skills from other repertoires (e.g., Receptive Language, Imitation, etc.) shortly after mastering these repertoires, highlights the possibility that one or more of these four specific repertoires may represent precursor skills required for further skill development. Moreover, given the language and learner skills associated with these repertoires (i.e., the spontaneous use of sounds and language, the motivation to comply with others and partake in learning tasks, the ability to generalize learned responses, and the ability to request for objects, events, or information, etc.), it follows that deficits in any of these skill areas may complicate the process of acquiring other important skills that typically develop at a later age (e.g., receptive language, labeling, imitation, etc.).

A second example illustrating that a complex relationship may exist across specific repertoires includes the finding that the Receptive Language and Labeling repertoires developed prior to the Intraverbal repertoire. Nonverbal stimuli present in the immediate, physical environment control a verbal labeling response (i.e., tact) whereas verbal stimuli control receptive and intraverbal responses (Skinner, 1957). In contrast to an intraverbal response, the use of receptive language requires one to emit a non-verbal response. As such, intraverbal responses require one to possess sufficient receptive language and labeling skills in order to accurately talk about things in their physical absence. Thus, a child that cannot respond to the words spoken by others and talk about objects or events in the immediate environment (i.e., tacts), would likely struggle to provide an accurate intraverbal response. This logic may explain why children acquired their receptive language and labeling skills prior to acquiring the skills from the Intraverbal repertoire.

Collectively, the aforementioned findings allow us to draw some general conclusions regarding typical skill development and yield some important implications for educational programming for individuals with ASD. First, we view skill development as a complex process in which specific $A B L L S-R$ repertoires may act as mediating or moderating variables that influence the age-related development observed across other $A B L L S-R$ repertoires. While the scope of our study does not enable us to confirm the accuracy of this conclusion, the developmental literature would benefit from further examining this topic. Second, when prioritizing teaching objectives for individuals with ASD, parents and professionals should initially teach and develop skills from the Basic Learner and Motor skills sections in the $A B L L S-R$ since these skills emerge and are mastered by typically developing children at a young age. Third, one 
should forego teaching academic skills to individuals with ASD until the learner displays mastery of other, more functional skills that typically occur earlier in development (e.g., skills from the Basic Learner and Motor skills sections). Finally, since typically developing children generally master the lower numbered $A B L L S-R$ items prior to the higher numbered items, parents and professionals should select learning objectives that target skills acquired at a younger age (i.e., lower numbered $A B L L S-R$ items) prior to teaching more advanced skills (i.e., higher numbered $A B L L S-R$ items). Given our data reveal some exceptions to this general finding, we encourage individuals to reference Tables 1 through 3 prior to selecting teaching objectives.

The present pilot study contains some limitations that warrant further discussion. First, we recruited a smaller than ideal participant sample given the number of levels of the independent variable that we examined. The use of a larger participant sample would lend further confidence to our findings and depending on the sample size, may lead to the establishment of normative data that documents the skills of a typically developing child at a given age. Because of this limitation alone, we view these findings as preliminary evidence of typical skill development that requires future research to confirm the accuracy of our findings (by using a larger participant sample and addressing the other limitations noted below).

A second and related limitation includes our age range used to examine typical skill development. Our data confirm that children do not master all of the skills from a few $A B L L S-R$ repertoires (e.g., Dressing) and sections (i.e., Academic and Self-help) by age six despite the increasing trends observed in our data. Using a larger age range that includes data from participants older than the age of six would enable researchers to identify when these skills fully develop.

A third limitation includes our decision to forgo the steps of calculating IOA data and obtaining measures of treatment fidelity. As we previously noted, we recruited assessors at various $A B L L S-R$ training seminars across the United States and Asia. This methodology complicated our ability to identify other, qualified individuals whom could collect IOA data and assess treatment fidelity throughout our lengthy data collection period. This limitation in particular hinders our ability to ascertain the extent that the assessors properly administered the $A B L L S-R$ and accurately scored the performance of the children. In light of this limitation, our method of recruiting participants ensured that all assessors received the same quality of training from the same individual (i.e., a Board Certified Behavior Analyst with over 35 years experience with conducting $A B L L S-R$ skills assessments) on how to administer the $A B L L S-R$ and collect data. Further, a recent study found that individuals without any formal training could administer the $A B L L S-R$ and still obtain highly reliable scores (Usry et al., 2017). While these facts may help to mitigate the effects of this limitation, improving upon the overall strength and believability of our results requires one to collect and interpret IOA data and to obtain measures of treatment fidelity. Taking these steps would represent one of several different strategies that researchers can include in future studies to further examine typical skill development.

Researchers can further our knowledge on typical skill development by addressing the noted limitations as well as some of the prevailing gaps in the literature. One strategy may include replicating the present study using a larger participant sample, collecting IOA data, and measuring treatment fidelity throughout the data collection period. Another related strategy could include collecting and analyzing data obtained from participants from different countries to better understand the generality of our results. In addition, researchers can shed further light on typical skill development by using a larger age range to more accurately pinpoint the age that children display mastery over more complex skills (i.e., various self-help and academic skills). A second area for future research includes determining the extent that complex relationships exist between the different $A B L L S-R$ repertoires. Specifically, future research may seek to examine whether some repertoires may function as mediating or moderating variables that influence or explain the age-related increases in skill development observed across other, potentially related repertoires. This area of research could yield invaluable information on which skill areas to target or prioritize given their relation to subsequent skill development.

\section{References}

Aman, M. G., Novotny, S., Samango-Sprouse, C., Lecavalier, L., Leonard, E., Gadow, K. D., \& Chez, M. (2004). Outcome measures for clinical drug trials in autism. CNS Spectrums, 9, 36-47. https://doi.org/10.1017/S1092852900008348

American Medical Association. (2014). CPT assistant. Chicago, IL: American Medical Association.

Ator, N. A. (1999). Statistical inference in behavior analysis: Environmental determinants? The Behavior Analyst, 22, 93-97. https://doi.org/10.1007/BF03391985

Center for Disease Control and Prevention (2018). Data and statistics. Retrieved from http://www.cdc.gov/NCBDDD/autism/data.html.

Fahmie, T. A., \& Hanley, G. P. (2008). Progressing toward data intimacy: A review of within-session data analysis. 
Journal of Applied Behavior Analysis, 41, 319-331. https://doi.org/10.1901/jaba.2008.41-319

Guldberg, K. (2010). Educating children on the autism spectrum: Preconditions for inclusion and notions of "best autism practice" in the early years. British Journal of Special Education, 37, 168-174. https://doi.org/10.1111/j.1467-8578.2010.00482.x

Leaf, R., \& McEachin, J. (1999). A work in progress: Behavior management strategies and a curriculum for intensive behavioral treatment for autism. New York: DRL Books.

Malkin, A., Dixon, M. R., Speelman, R. C., \& Luke, N. (2016). Evaluating the relationships between the PEAK Relations Training System-Direct Training Module, Assessment of Basic Language and Learning Skills-Revised, and the Vineland Adaptive Behavior Scales-II. Journal of Developmental and Physical Disabilities. Advance online publication. https://doi.org/10.1007/s10882-016-9527-8

Partington, J. W. (2010a). The Assessment of Basic Language and Learning Skills-Revised. Pleasant Hill, CA: Behavior Analysts, Inc.

Partington, J. W. (2010b). The Assessment of Basic Language and Learning Skills-Revised: Scoring instructions and IEP development guide. Pleasant Hill, CA: Behavior Analysts, Inc.

Partington, J. W., Bailey, A., \& Partington, S. P. (2016). A pilot study examining the test-retest and internal consistency reliability of the ABLLS-R. Journal of Psychoeduational Assessment. Advance online publication. https://doi.org/10.1177/0734282916678348

Perone, M. (1999). Statistical inference in behavior analysis: Experimental control is better. The Behavior Analyst, 22, 109-116. https://doi.org/10.1007/BF03391988

Schwartz, I. S., Boulware, G. McBride, B. J., \& Sandall, S. R. (2001). Functional assessment strategies for young children with autism. Focus on Autism and Other Developmental Disabilities, 16, 222-227. https://doi.org/10.1177/108835760101600404

Sidman, M. (1960). Tactics of scientific research. New York, NY: Basic Books

Skinner, B. F. (1957). Verbal behavior. New York: Appleton-Century-Crofts. https://doi.org/10.1037/11256-000

Sparrow, S. S., Cicchetti, D. V., \& Balla, D. A. (2005a). Vineland adaptive behavior scales (2nd ed.). Bloomington, MN: Pearson.

Sparrow, S. S., Cicchetti, D. V., \& Balla, D. A. (2005b). Vineland adaptive behavior scales: Survey forms manual (2nd ed.). Bloomington, MN: Pearson.

Sundberg, M. (2008). VB-MAPP Verbal Behavior Milestones Assessment and Placement Program: A language and social skills assessment program for children with autism or other developmental disabilities: Guide. Concord, CA: AVB Press.

Thompson, T. (2011). Individualized autism intervention for children. Baltimore, MD: Paul H. Brookes Publishing Co.

Usry, J. N., Partington, S. W., \& Partington, J. W. (2017). Using expert panels to examine the content validity and inter-rater reliability of the ABLLS-R. Journal of Developmental and Physical Disabilities, 1, 27-38. https://doi.org/10.1007/s10882-017-9574-9

\section{Copyrights}

Copyright for this article is retained by the author(s), with first publication rights granted to the journal.

This is an open-access article distributed under the terms and conditions of the Creative Commons Attribution license which permits unrestricted use, distribution, and reproduction in any medium, provided the original work is properly cited. 